\title{
Neurophotonics
}

\section{Route to genetically targeted optical electrophysiology: development and applications of voltage-sensitive fluorescent proteins}

Walther Akemann

Chenchen Song

Hiroki Mutoh

Thomas Knöpfel 


\title{
Route to genetically targeted optical electrophysiology: development and applications of voltage-sensitive fluorescent proteins
}

\author{
Walther Akemann, ${ }^{\mathrm{a}, \mathrm{b}}$ Chenchen Song, ${ }^{\mathrm{a}}$ Hiroki Mutoh, ${ }^{\mathrm{c}}$ and Thomas Knöpfel ${ }^{\mathrm{a}, *}$ \\ Imperial College London, Department of Medicine, London W12 ONN, United Kingdom \\ IInstitute of Biology, CNRS UMR 8197, École Normale Supérieure, 46 rue d'Ulm, 75005 Paris, France \\ 'Hamamatsu University School of Medicine, Department of Neurophysiology, Shizuoka 431-3192, Japan
}

\begin{abstract}
The invention of membrane voltage protein indicators widens the reach of optical voltage imaging in cell physiology, most notably neurophysiology, by enabling membrane voltage recordings from genetically defined cell types in chronic and life-long preparations. While the last years have seen a dramatic improvement in the technical performance of these indicators, concomitant innovations in optogenetics, optical axon tracing, and high-speed digital microscopy are beginning to fulfill the age-old vision of an all-optical analysis of neuronal circuits, reaching beyond the limits of traditional electrode-based recordings. We will present our personal account of the development of protein voltage indicators from the pioneering days to the present state, including their applications in neurophysiology that has inspired our own work for more than a decade. $\odot$ The Authors. Published by SPIE under a Creative Commons Attribution 3.0 Unported License. Distribution or reproduction of this work in whole or in part requires full attribution of the original publication, including its DOI. [DOI: 10.1117/1.NPh.2.2.021008]
\end{abstract}

Keywords: optics; photonics; voltage imaging; voltage-sensitive fluorescent proteins; cortex; electroencephalogram; brain rhythms. Paper 14074VSSR received Oct. 23, 2014; accepted for publication Dec. 11, 2014; published online Jan. 14, 2015.

\section{Introduction}

In this volume of Neurophotonics, much will be said about the inspiration and success of the early quest by Cohen and coworkers more than 50 years ago, ${ }^{1}$ for optical signals to report electrical activity from excitable membranes. It was through this seminal work that the idea of optical recording of membrane electrical activity of cells and across cellular networks was born, initiating the development of optical functional imaging methods in neurophysiology. ${ }^{2-4}$ Given the universal role of the optical microscope to visualize cellular structure and diversity in every domain of biology, it is easy to imagine the appeal this work had on generations of students and researchers, attracting many to devote their research careers to the optical imaging of neural function. While the development of an all-optical approach to electrophysiology continues, it is evident that the genealogy of this field roots in the early work of Cohen and coworkers starting in the 1960s during the golden era of membrane biophysics.

Since the discovery by Cohen and coworkers of Merocyanine-540, the first synthetic dye membrane voltage indicator, ${ }^{5}$ enormous efforts have been undertaken to identify new chemical compounds suitable as fluorescence probes of membrane voltage with improved sensitivity, reduced toxicity, and diverse spectral properties, ${ }^{6-12}$ enabling myriads of applications in general physiology and neurophysiology. ${ }^{13,14}$ Following the invention of voltage-sensitive dye imaging, indicators for intracellular calcium concentration later emerged as a practical alternative to probe spiking events in neurons by exploiting the large rise of intracellular calcium concentration associated with action potential discharge. ${ }^{15-18}$ Although optical calcium

*Address all correspondence to: Thomas Knöpfel, E-mail: t.knopfel@imperial .ac.uk measurements generally achieve significantly better signal-tonoise ratio (SNR) for action potential detection at the cellular resolution in two-photon imaging in vivo, limitations exist: first, given the intrinsic slowness of the intracellular calcium dynamics as opposed to the much faster membrane voltage change, spike times cannot be inferred with a temporal precision compatible with classical electrophysiological recordings. For the same reason, a reliable distinction of single spikes can be impaired if spikes are followed with a short delay, as in highfrequency spike trains or spike bursts. Second, events that do not reach the firing threshold evoke no (e.g., hyperpolarizing synaptic potentials) or vanishingly small (subthreshold excitatory synaptic potentials) calcium transients.

In principle, voltage imaging is better suited to probe the entirety of cellular electrical signals that define the language of the nervous system, including excitatory and inhibitory synaptic potentials, action potentials in single cells, as well as the associated population signals [local field potentials and the electroencephalogram (EEG)]. ${ }^{19,20}$ Today, voltage imaging using small molecular weight voltage-sensitive dyes is an established method in neurophysiology for probing population membrane potential dynamics on the mesoscopic scale (millimeters of field of views ${ }^{13,21-23}$ ). At the subcellular level, voltage-sensitive dye imaging was applied to voltage signals in fine dendritic branches, spines, and axons. ${ }^{24,25}$ Cellular level voltage imaging in intact mammalian brain has been technically very challenging. ${ }^{26}$ The principal limitations of low-weight voltage-sensitive dyes remain their immanent, pharmacological side effects, ${ }^{27}$ toxicity and phototoxicity, indiscriminate staining of neuronal and glial membranes, small signal amplitude, impossibility to target specific cells, and fugacity of dye staining impeding long-term imaging in vivo. ${ }^{19}$

The development of genetically encoded voltage indicators (GEVIs) over the last decade was animated by the idea to 
overcome these limitations. ${ }^{28}$ While the GEVI class of voltage indicators is still rapidly growing, several GEVIs were already benchmarked for practical use in vivo. In the following sections, we will summarize the history and the present state of the development of these indicators together with the emerging applications of GEVIs in neurophysiology research.

\section{History of Genetically Encoded Voltage Indicator Development (1997 to 2012)}

At the time of the cloning of green fluorescent protein (GFP) by Douglas Prasher in the early 1990s, traditional low-weight voltage-sensitive dyes and calcium indicators were already well established for imaging neuronal electrical activity. ${ }^{29,30}$ However, the discovery of GFP's heterologous expression ${ }^{31}$ and the subsequent development of its color variants opened the possibility of a fundamentally different class of fluorescence indicators that are entirely built from proteins and fully encoded in DNA.

The translation of traditional synthetic fluorescence indicators into protein space was, however, everything but straightforward. Organic fluorescence indicators are engineered to employ optical transitions, whose transition energy or strength is influenced by extramolecular parameters such as the local electrical field or ligand binding. In dyes, these interactions are usually reliant on delocalized valence states, as these states are highly sensitive to changes of the inner-molecular electrostatic field. In proteins, on the other hand, valence states are spatially much more constrained as a consequence of the much larger molecular size. The first genetically encoded calcium indicators were therefore designed, with a steric rather than an exclusively electronic mechanism in mind, as a construct where a cyan and a yellow fluorescent protein (FP) are, respectively, placed at the $\mathrm{N}$ - and C-terminal positions of a calmodulin-M13 calcium-binding domain. ${ }^{32,33}$ It was speculated that upon calcium chelation, the conformational contraction of calmodulin-M13 would alter the steric distance between the two FPs. This would in turn manifest optically as a change of the electromagnetic exited state energy transfer from cyan to yellow FP via the steep distance-dependence of Förster resonance energy transfer (FRET) between the chromophores within the range of the nonradiative optical near field. ${ }^{34}$ While the combination of a calcium-binding domain and a fluorescence reporter in Cameleon and other GECIs still reminds of the same structural dichotomy in low-molecular weight calcium indicators (e.g., Fura2 or Oregon Green BAPTA), the creation of the first protein voltage indicators was inspired by the conformational changes that control the opening and closing of voltage-gated ion channels. In 1997, Siegel and Isacoff ${ }^{35}$ reported a fusion protein denoted as FlaSh, which consisted of a shaker voltage-gated potassium channel with a GFP fused to its C-terminus (Fig. 1). FlaSh is considered as the first GEVI and was based on the assumption that the voltage-dependent gating of an ion channel, involving conformational transition between the conducting and the nonconducting states of the channel, could be exploited to actuate a fluorescence reporter in a manner resembling the fluorescence modulation observed from channel-conjugated organic dyes known to be able to report conformational changes in ion channels. ${ }^{36,37}$ Detailed analysis later, however, suggests that FlaSh is a reporter of C-type inactivation, rather than gating, of the shaker voltage-gated potassium channel. ${ }^{35}$

Following this idea, but introducing GFP into a skeletal muscle sodium channel instead, led to another prototypic
GEVI construct, named SPARC ${ }^{38}$ (Fig. 1). A very different concept of a GEVI, named VSFP1 (voltage-sensitive fluorescent protein; Fig. 1), was introduced by our lab (Knopfel lab at RIKEN, Japan); ${ }^{39}$ slightly earlier than SPARC. VSFP1 differed from FlaSh and SPARC in three major aspects: While voltage-gated potassium channels (Kvs) consist of four subunits each containing six transmembrane segments (S1-S6), VSFP1 included only transmembrane segments S1-S4 (that form the putative voltage sensor domain) of a single Kv2.1 potassium channel subunit. Second, directly linked to the end of the S4 transmembrane segment of the Kv2.1 voltage sensor domain VSFP1, carried a pair of cyan and yellow FPs conceived as a FRET reporter for voltageactivated conformational transitions of the S1-S4 voltage sensor. Third, VSFP1 was designed as a dual emission (ratiometric) indicator to help abridge movement artifacts and hemodynamic modulation of optical signals in in vivo imaging. While FlaSh, VSFP1, and SPARC were successful as proofof-principle when tested in Xenopus oocytes, they performed poorly in mammalian neurons, presumably due to faint membrane expression and strong accumulation in internal membranes and organelles including the endoplasmic reticulum (ER) and the Golgi apparatus. ${ }^{40}$ Many years of efforts to improve GEVI export from ER and trafficking to plasma membranes remained fruitless and helped to cast doubts on the concept. In particular, the "voltage-sensor-domain-only" concept of VSFP1 faced criticism citing the lack of proof that an isolated ion channel voltage sensor can function as a functionally independent domain.

While the search for improvements of GEVI membrane targeting mostly focused on channel trafficking signals, a solution eventually emerged from the discovery of voltage sensor-containing phosphatase in Ciona intestinalis (Ci-VSP) by Okamura and coworkers. Ci-VSP features a voltage sensor that resembles the S1-S4 voltage sensing domain of Kv channels. However, instead of operating an ion channel, the Ci-VSP voltage sensor controls the activity of an intracellular phosphatase. ${ }^{41} \mathrm{Ci}-\mathrm{VSP}$ was discovered by genomic data mining as it preserves the crucial sequence motives that define ion channel voltage sensors across species. Yet, it was the first time that a native voltage sensor was found in a protein other than an ion channel. The Ci-VSP discovery was of importance for the development of GEVIs for at least three reasons. First, it provided strong support for the view of the voltage sensor as an independent functional domain that has motivated the design of VSFP1, a conclusion that later was corroborated by other observations, notably the discovery of the voltage-sensor-only proton channel Hv1. ${ }^{42,43}$ Second, even more importantly, in contrast to ion channels that require assembly into tetrameric superstructures to acquire plasma membrane localization and function, there was no evidence for oligomerization as a necessary condition for Ci-VSP function. ${ }^{44}$ To ensure correct channel assembly in the ER, ion channels are thought to carry ER retention signals that become masked by successful tetramerization before trafficking to the membrane. ${ }^{45}$ The possible existence of such unmasked retention signals was suspected as an impediment for efficient membrane trafficking of the first generation GEVIs. And third, because of the phylogenic distance between $C$. intestinalis and mammals, it seemed possible that even if ER retention signals were present in Ci-VSP they might not be recognized efficiently by mammalian cells. Based on these considerations, we began to generate a set of constructs based on the VSFP1 template, but with the Kv2.1 


\section{GEVI design concepts based on FPs inserted into ion channels}

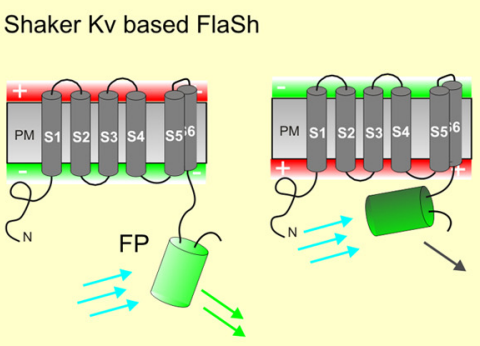

SPARC

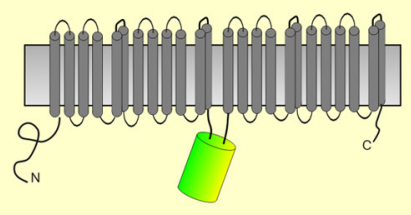

FlaSH split FP

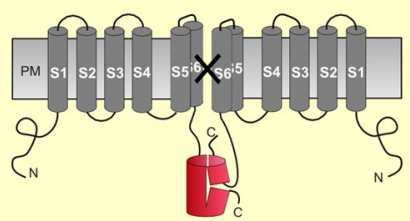

Isolated voltage sensing domain based VSFPs and similar concepts

FRET based VSFP1/2s
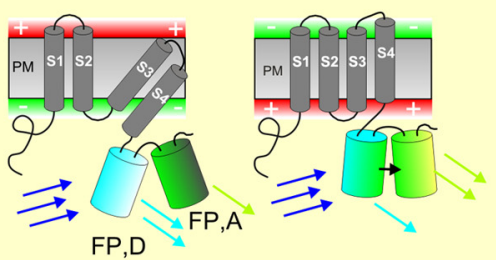

FP,D

FRET based VSFP butterflies
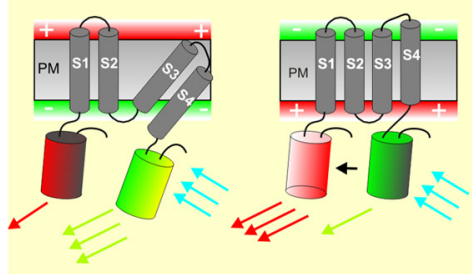

single FP based VSFP3s

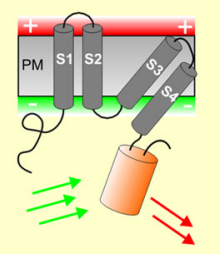

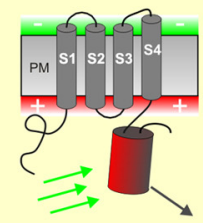

chimeric VSFP butterflies

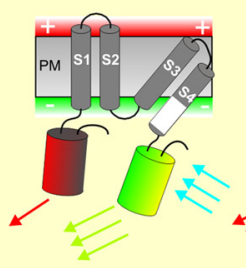

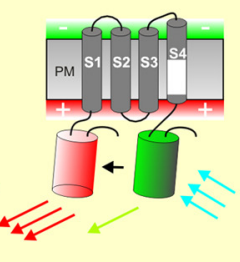

ASAP1
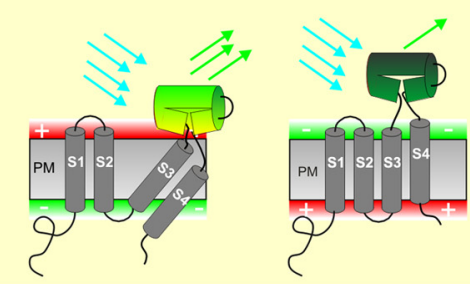

\section{Opsin based GEVI designs}

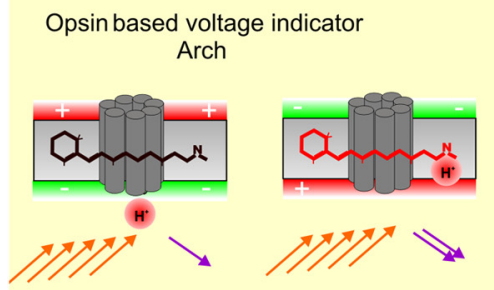

opsin/FRET voltage probe

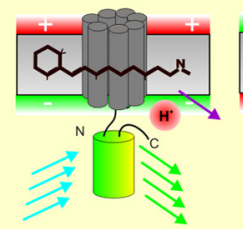

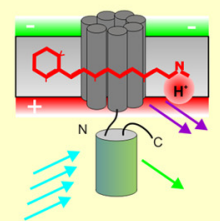

cpFP based VSFPs

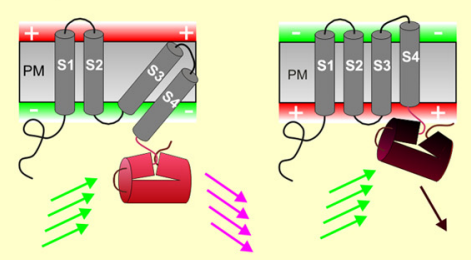

\section{Upcoming concepts}

Membrane integrated electrochromic FP

FP dimerisation based sensors

Etc

Fig. 1 Overview of genetically encoded voltage indicator (GEVI) molecular designs: Upper row shows the GEVIs based on the insertion of fluorescent proteins (FPs) (depicted as barrels) into complete ion channel proteins with segments (e.g., S1-S6) that cross the plasma membrane (PM). In the FlaSh-type voltage indicator, a FP is fused into the $\mathrm{C}$-terminal portion of a Shaker potassium channel subunit. Tetramers of subunits form a channel structure which is made nonconducting by a point mutation. Modulation of FlaSh fluorescence is triggered by voltage-dependent rearrangements, probably corresponding to channel C-type inactivation. Middle panels show the GEVIs based on isolated voltagesensing domains. In Förster resonance energy transfer (FRET)-based voltage-sensitive probes of the voltage-sensitive fluorescent protein (VSFP1/2) type, the voltage-sensor domain, consisting of four segments (S1-S4), is fused to a pair of FPs (FP, D: FRET donor; FP, A: FRET acceptor). A change in membrane potential induces a rearrangement of the two FPs that is optically reported as a change in the ratio between donor and acceptor fluorescence. Single FP and circularly permuted (cp) FP probes of the VSFP3 family are monochromatic. In FRET-based voltage-sensitive probes of the VSFP Butterfly family, the voltage-sensor domain is sandwiched between two FPs. Lower panels show the GEVIs based on opsins. A change in membrane potential induces increased fluorescence of the retinal molecule. The microbial rhodopsin-based voltage indicator Arch shows an increased fluorescence of the retinal molecule when the membrane potential is increased. In opsin/FRET probes, a FP in quenched by retinal in a voltage-dependent manner. 
voltage sensor being replaced by that of Ci-VSP. Notably, the very first construct (VSFP2a-d) displayed dramatically improved membrane localization compared with VSFP1, ${ }^{46,47}$ while at the same time showing a significant voltage report in mammalian cells. However, the voltage sensitivity was seen only at membrane potentials far above normal membrane potential fluctuations in mammalian cells. This was not a surprise since the Ci-VSP voltage sensor was known to activate at very positive voltages. ${ }^{41}$ This problem was solved by mutagenesis of the voltage sensor in a region of the S4 voltage sensor segment resulting in a charge-neutalizing mutation R217Q of a polar S4 arginine that shifted the Ci-VSP voltage sensor activation to negative potentials within the dynamic range of the neuronal electrical signaling. ${ }^{47}$ Interestingly, this charge neutralization did not alter the total effective sensing charge available to the voltage gate of 1.2 elementary charges. ${ }^{48}$ For the historic perspective taken here, we like to note that the prepublication sharing of these results with Cohen and colleagues provided preliminary data for the successful acquisition of the first multimillion NIH grant on the development of GEVIs in 2006. Further optimization by tailoring the peptide linker between the voltage sensor and the FP reporter of VSFP2.1 eventually produced VSFP2.3. ${ }^{48}$ The VSFP2s were the first GEVIs to overcome the membrane trafficking impediment characteristic of the first GEVI generation and were the first GEVIs to exhibit convincing optical voltage report in the neuron-like PC12 cells, ${ }^{47,48}$ mouse primary neuronal culture, acute cortical brain slices, and mouse cortex in vivo. ${ }^{49}$ The VSFP2s were later extended into a larger family by several GEVI constructs engineered as fusions of the Ci-VSP voltage sensor with redshifted FRET reporters, notably Mermaid, ${ }^{50}$ VSFP2.4, ${ }^{51}$ and VSFP-CR. ${ }^{52}$

In addition to overcoming the many years of deadlock in GEVI development, the VSFP2s also served as the first model for studies of the biophysical mechanism of GEVI function. Since the transition between the ion channel resting and activated conformations is driven by movable charges within the voltage sensor that will translocate between equilibrium positions as a function of membrane voltage,${ }^{53}$ sensor activation gives rise to a measurable extracapacitive current, named the sensing or gating current. This current is generated by the displacement of an ionic screening charge at the external membrane interface following translocation of the sensing charge within the membrane. ${ }^{54}$ Simultaneous fluorometric and sensing current measurements from VSFP2.3 expressed in Xenopus laevis oocytes ${ }^{55}$ and in PC12 cells ${ }^{48}$ under voltage clamp revealed a two-step activation process of the VSFP2.3 fluorescence response, with a fast time component reflecting the transfer of sensing charge and a subsequent component with slower time course and little associated charge transfer. ${ }^{56}$ At this stage, it came as a fortunate surprise that the measured kinetics of VSFP2.3 activation turned out to be faster in PC12 cells than in $X$. oocytes, as manifested by a fast activation $\mathrm{ON}$ time constant of $3 \mathrm{~ms}$ in PC12 cells at $25^{\circ} \mathrm{C}^{56}$ versus $20 \mathrm{~ms}$ in oocytes. ${ }^{55}$ At $35^{\circ} \mathrm{C}$ (that is closer to the physiological body temperatures of rodents and humans), the fast activation $\mathrm{ON}$ time constant of VSFP2.3 is $2 \mathrm{~ms}^{51}$

While a slow component of fluorescence activation contributes significantly to the overall VSFP2.3 fluorescence response in the steady state, it is the fast-activating initial component that dominates the dynamic VSFP response on the time scale of fast neuronal voltage transients. ${ }^{56,57}$ The contribution of the fast component relative to the slower component was significantly improved with the development of VSFP2.4s and VSFP $3 \mathrm{~s}^{48,49,51,58}$ and other similar voltage sensing domainbased probes described below.

While VSFP2.3 and VSFP2.4 were the first GEVIs to be successfully tested in the mouse brain in vivo, it was obvious that further SNR improvement and kinetics acceleration were required to enable applications such as reliable detection of somatic action potentials in single neurons. ${ }^{49}$ The search for improved GEVIs explored a variety of ideas leading to GEVI constructs that employed voltage sensors from non-Ciona VSPs, ${ }^{59}$ GEVIs that used the Ci-VSP voltage-sensor together with a single FP reporter (dubbed VSFP3s) 48,58,60 $^{\text {or a circu- }}$ lar-permuted $\mathrm{FP},{ }^{61,62}$ split FPs into two half FPs inserted randomly into the Shaker potassium channel, ${ }^{63}$ and a GEVIturned archaerodopsin named $\operatorname{Arch}^{64,65}$ (Fig. 1). In this series of constructs, Arch was perhaps the most surprising one due to its unique mechanism in the absence of an ion-channeltype of voltage sensor, but involving a modulation of fluorescence caused by a voltage-sensitive proton transfer in the transient excited state of the retinal chromophore. ${ }^{66}$ Voltagesensor-based GEVIs exhibit a sigmoidal fluorescence-voltage relationship consistent with a voltage-activated transition between the activated and the resting states of the voltage sensor, but Arch featured a more linear relationship and large voltage sensitivity. Unfortunately, the fluorescence quantum yield of the retinal chromophore in Arch is extremely low $(\sim 1 / 1000$ compared with GFP), resulting in an output signal that is easily disturbed by background fluorescence ${ }^{67}$ and the requirement of high-illumination intensities may cause considerable heating of the preparation.

Reengineering of VSFP2.4 eventually led to a new GEVI, named VSFP Butterfly, with largely enhanced performance. ${ }^{68}$ In VSFP Butterfly-1.2, the mKate2 acceptor FP is moved from the C-terminal (as in VSFP2.4) to a position at the $\mathrm{N}$-terminus, thus yielding a configuration where the two FPs are flanking the voltage sensor (Fig. 1). While VSFP Butterfly-1.2 inherited its fast activation kinetics from VSFP2.4, its steady-state activation is shifted closer to the neuronal resting potential, characterized by a half activation voltage of $-70 \mathrm{mV}$, versus $-50 \mathrm{mV}$ in VSFP2.4, together with a larger weight of the fast component of fluorescence response. ${ }^{68}$ This feature, together with the insertion of an ER export motif to enhance trafficking to the membrane, ${ }^{69,70}$ greatly improved the sensitivity to subthreshold voltage transients, but also permitted the detection of fast action potentials in pyramidal cells in single trials. ${ }^{68}$ The Butterfly design was later reproduced in Mermaid-2, using a cyan FP at the N-terminal and a yellow FP at the C-terminal position of the Ci-VSP voltage sensor, ${ }^{71}$ a construction that finally overcame the poor neuron membrane localization of Mermaid. ${ }^{72}$ At the same time, another GEVI called ArcLight was introduced using the $\mathrm{pH}$-sensitive super ecliptic pHluorin with a A227D mutation as a single FP reporter attached to the Ci-VSP voltage sensor, ${ }^{60}$ a principle that was first realized in the VSFP3 series of indicators. ${ }^{48,58}$ ArcLight has a large steady-state dynamic range of $35 \%$ together with modest kinetics but can report broad action potentials in cultured neurons with high $\mathrm{SNR}^{60}$ and action potentials and synaptic potentials in local neuronal circuits of Drosophila melanogaster in vivo. ${ }^{73}$ 


\section{Current Trends in Genetically Encoded Voltage Indicator Development (2012 to 2014)}

The remarkable boost in GEVI performance over the last years was accompanied by a steady increase in the number of available GEVI constructs (see Fig. 1) calling for a systematic approach to quantify the performance of each GEVI under experimental conditions that represent their main application. While indicator performance is partly predictable from biophysical parameters, including extinction coefficient, emission quantum yield, rate constant of photobleaching, total effective sensing charge, half activation voltage, and $\mathrm{ON}$ and $\mathrm{OFF}$ time constants, ${ }^{19,56}$ it also depends on characteristics of the expression system comprising the biochemical identity of target cells, method of gene delivery, transcription regulation and trafficking mechanisms in target cells, altogether affecting expression level, surface density, and level of background fluorescence. The perhaps biggest hurdle on the route toward a broadly applicable "optogenetic" approach to electrophysiol$\mathrm{ogy}^{74}$ results from the difficulty to track high-frequency content in the power spectrum of neuron electrical signals. While traditional electrophysiological recordings permit cut-off frequencies to the $\mathrm{MHz}$ range at $100 \mathrm{kHz}$ sampling rates or faster, the early generation of GEVIs had a cut-off well below $100 \mathrm{~Hz}{ }^{68}$ although optical sampling at much higher rates is technically feasible. Therefore, increasing GEVI bandwidth and SNR remains a central objective for the development of next generation GEVIs. To this end, several new designs aiming to accelerate GEVI kinetics were recently developed. Our lab pursued the idea to substitute parts of the voltage gate of the Ci-VSP voltage sensor with homologous components of the fast-gating Kv3.1 potassium ion channel. ${ }^{75}$ By establishing a library of VSFP constructs with chimeric Ci-VSP/Kv3.1 voltage sensors introduced into the VSFP2.3 template, we observed modest kinetic enhancements when replacing parts of the S4 region. Larger replacements in regions including S4, S3, S2, and part of the S1-S2 extracellular loop resulted in significantly accelerated $\mathrm{ON}$ and $\mathrm{OFF}$ kinetics, equivalent to time constants faster than $2 \mathrm{~ms}$, and a complete annihilation of the slow secondary activation step characteristic of the Ci-VSP sensor. We also created a C5 chimeric mutant with part of the S4 transplanted from Kv3.1, and a Butterfly version where the acceptor $\mathrm{FP}$ in $\mathrm{C} 5$ is moved from the $\mathrm{C}$ to the $\mathrm{N}$ terminus ${ }^{76}$ mimicking the FP configuration of the nonchimeric VSFP Butterfly. The chimeric C5 VSFP Butterfly mutant exhibited similar fast kinetics as C5 together with a negatively shifted activation ${ }^{76}$ in agreement with a similar shift in nonchimeric VSFP Butterfly ${ }^{68}$ Compared with VSFP Butterfly-1.2, C5 chimeric VSFP Butterfly features a much faster OFF transition resulting in improved total bandwidth and the ability to report voltage commands up to $200 \mathrm{~Hz}$ as demonstrated in cell culture. ${ }^{76}$

Meanwhile Adam Cohen and coworkers developed a second generation version of Arch making use of the intrinsically fast photoactivation kinetics of archaerodopsins and searching for brighter mutants by random mutagenesis combined with fluorometric screening. ${ }^{77}$ This work resulted in two new rhodopsin-based GEVIs named QuasAr1 and QuasAr2, improving the low quantum yield of Arch by an order of magnitude. Most of all these variants can achieve fast GEVI kinetics in the absence of accompanying large photocurrent unlike Arch. With ON and OFF time constants faster than $100 \mu$ s at room temperature, ${ }^{77}$ QuasAr1 is the fastest GEVI reported so far and thus the first GEVI to be able to map the waveform of a fast somatic action potential in a manner fully compatible with classical intracellular electrophysiological recording. ${ }^{77}$ However, in spite of some improvement in brightness and membrane localization from Arch, QuasAr1 and QuasAr2 remain dim compared with GEVIs using high-yield FP reporters, and their untested in vivo reporting ability may still suffer from insufficient SNR.

A possibility to overcome the low brightness of Arch-type GEVIs was recently explored in the lab of Mark Schnitzer, as well as independently in the labs of Robert Campbell and Adam Cohen. ${ }^{78-80}$ The basic idea was to fuse a high-quantum yield FP to Arch-type GEVIs, so that the FP can act as a FRET donor to the retinal rhodopsin chromophore (Fig. 1). This approach yielded MacQ-mCitrine, employing a Mac rhodopsin in lieu of Arch and showing a fast activation time constant of $2.8 \mathrm{~ms}$, large dynamic range, and significant expression at the cell surface. ${ }^{81}$ As the MacQ-FRET GEVI owes its brightness to the mCitrine donor and its photon yield is compatible with other FP-based GEVIs, in vivo testing in the mouse cerebellum demonstrated the probe's ability to report broad $(\sim 10 \mathrm{~ms})$ calcium spike-induced regenerative potentials in Purkinje cell dendrites. ${ }^{81}$ Similar GEVIs were created using QuasAr2 instead of MacQ as the FRET acceptor, ${ }^{79}$ where efficient linkage of QuasAr2 to a C-terminal FP reporter within the distance of the Förster radius of the retinal/FP reporter gives rise to functional and bright indicators. However, when compared with QuasAr-2 itself, these GEVIs tend to show lower dynamics and slower kinetics, including increased contribution of slow activation. $^{79}$

Motivated by the quest for speedy GEVI kinetics, ASAP1 is a GEVI design developed by Michael Lin and coworkers. ${ }^{82}$ ASAP1 was constructed from the voltage sensor of Gallus gallus voltage-sensing phosphatase (Gg-VSP) by inserting permuted enhanced GFP into the S3-S4 extracellular loop, after attempts to insert the reporter at a similar position to the $\mathrm{Ci}$ VSP voltage sensor failed to yield sufficient surface localization. ${ }^{82}$ ASAP1 differs from previous single FP-GEVIs that had been placing the FP reporter at the $\mathrm{C}$-terminus of the $\mathrm{Ci}$ VSP voltage sensor ${ }^{58,60}$ and is the first GEVI featuring an extracellular reporter with good surface localization, large dynamic range, and fast kinetics, yielding a fast time constant of $2.1 \mathrm{~ms}$ at room temperature. ${ }^{82}$

The ongoing development of enhanced GEVI variants apparently has established several successful design principles with leading constructs reaching voltage sensitivity and kinetics in par with the best performing VSDs, as judged from the available cell culture assays. Even though caution should be in place as careful comparative studies of GEVI variants are still missing, this is a remarkable progress. The GEVI function will necessarily be sensitive to the biochemical conditions in their host cells and to the method of gene transfer applied, creating complexity unknown to VSD staining procedures. For these reasons, validation of GEVI function and SNR (e.g., signal detectability) in in vivo imaging recordings remains a necessary step on the route to successful application of GEVIs in optical neurophysiology.

\subsection{Application of VSFPs to Cortical Functional Imaging In Vivo}

We tested VSFP2.3, VSFP2.4, VSFP Butterfly-1.2, and chimeric VSFP Butterfly for transcranial cortical imaging in the 
mouse cortex. ${ }^{49,76,83}$ Using in utero electroporation of pCAGVSFP plasmids into neuroepithelial cells of the subventricular zone at embryonic age E15.5, we obtained indicator expression limited to a sparse population of pyramidal cells in layer $2 / 3$. Simultaneous measurement of donor and acceptor emissions allowed efficient elimination of hemodynamic noise caused by the pulsatile blood flow and presented as a correlated signal at heart beat frequency in both imaging channels supplied by the

(a)

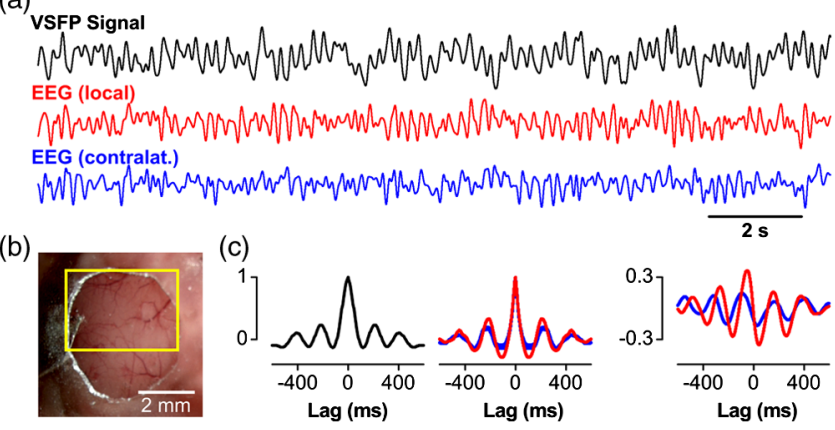

Fig. 2 Comparison of VSFP recording and cortical electroencephalogram (EEG): (a) VSFP Butterfly-1.2 signal (black) recorded from a large region of interest (ROI) over somatosensory cortex [see (b)] along with EEG signals from ipsilateral (red) and contralateral cortex (blue) showing spontaneous rhythmic cortical activity of a mouse under deep pentobarbital anesthesia. Signals are low-pass filtered with $10-\mathrm{Hz}$ cutoff. (b) Optical window for transcranial voltage imaging of somatosensory cortex. The yellow rectangle marks the ROI used to extract the VSFP signal shown in (a). (c) Autocorrelations of VSFP (left) and EEG signals (middle) and cross-correlations (right) of the VSFP signal [black trace in (a)] with ipsilateral (red) and contralateral (blue) EEG signals. Data adapted from Ref. ${ }^{68}$
mCitrine/mKate2 FRET reporter of Butterfly. ${ }^{83}$ Elimination of hemodynamic noise is a significant advantage afforded by dual-emission reporters, such as FRET reporter, as real-time or off-line hemodynamic noise cancellation ${ }^{83}$ significantly improves SNR in in vivo recordings. ${ }^{84,85}$ By recording sensory responses in the whisker barrel cortex in anesthetized animals under similar conditions, we found that the recordings from VSFP Butterfly-expressing animals offered consistently better SNR than those expressing VSFP2.3 or VSFP2.4. This is consistent with in vitro characterization of the indicators. Voltageclamped Butterfly-expressing pyramidal neurons in vitro exhibited faster voltage responses due to a larger weight of the fast response step and an activation onset at more negative potentials. ${ }^{83}$

Given these properties, we reasoned that the Butterfly GEVIs should excel in high-resolution optical readout of electroencephalographic signals (EEG) across cerebral cortex. To show this, we optically recorded slow-wave activity in anesthetized animals and compared the VSFP signal acquired from a transcranial optical window over somatosensory cortex [Fig. 2(b)] with simultaneously recorded ipsilateral and contralateral single-channel-EEG [see Fig. 2(a)]. These signals reported the same slow-wave periodicity of 2 to $5 \mathrm{~Hz}$ as revealed by similar VSFP and EEG autocorrelations, as well as periodic cross-correlation [Fig. 2(c)], which is markedly stronger for the local EEG [Fig. 2(c)]. After optimizing the transfection protocol using multiple rounds of plasmid electroporation, it was possible to achieve nearly homogeneous VSFP expression across the cortical hemisphere enabling hemisphere-wide VSFP imaging [Fig. 3(a), bottom]. Presentation of a tactile stimulus to the contralateral $\mathrm{C} 1$ whisker in an anesthetized animal [Fig. 3(a), top] elicited the propagation of the Butterfly-reported sensory-evoked signal at a velocity of 0.1 to $0.05 \mathrm{~m} / \mathrm{s}^{83}$ (a)

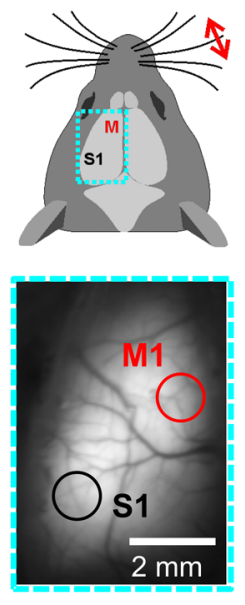

(b)

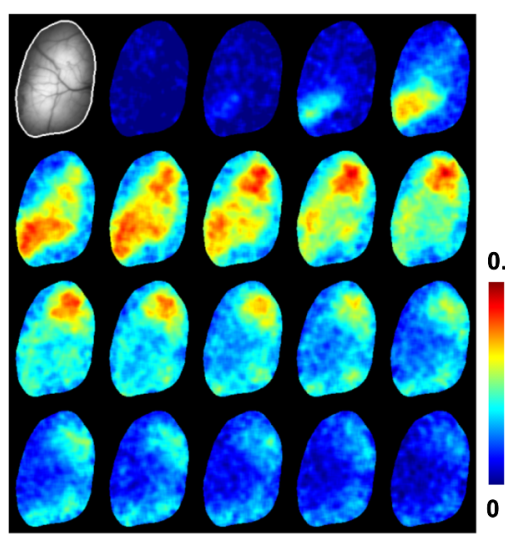

(c)

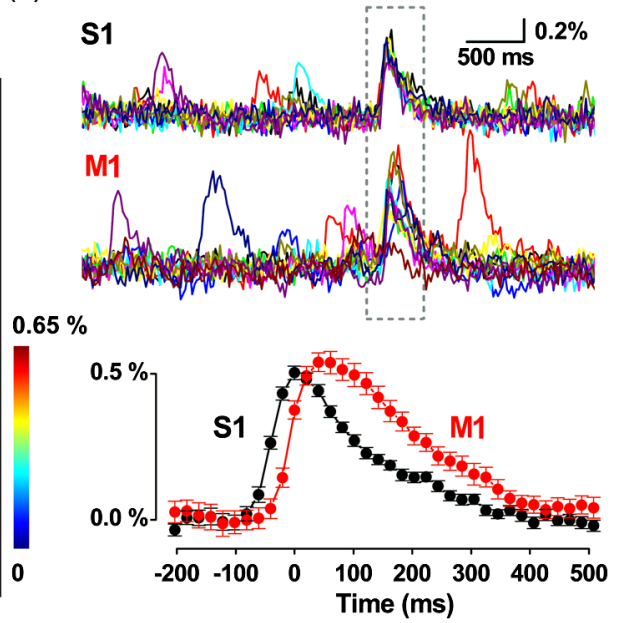

Fig. 3 Whole hemisphere VSFP imaging of sensory-evoked and spontaneous cortical electrical activity: (a) Top: schematic dorsal view of the mouse head with imaging region of the left cortical hemisphere indicated as dotted rectangular (cyan). Donor (mCitrine) and acceptor (mKate2) were recorded with two synchronized CCD cameras at 50 frames/s in $4 \mathrm{~s}$ trials with $30 \mathrm{~s}$ intertrial interval with a tactile stimulus (single deflection) delivered to the contralateral C1 whisker via a piezo drive triggered by the imaging clock after $2 \mathrm{~s}$ baseline recording. Bottom: transcranial mCitrine fluorescence image of the left cortical hemisphere. (b) Poststimulus, single-trial time lapse sequence (20 ms interval) of $\Delta R / R_{0}$ images after spatial filtering with a $2 \times 2$ Gauss filter. The first image (gray scale) shows the mCitrine baseline image with pixels outside of the optical window masked. (c) Top: time courses of 25 consecutive trials of primary sensory (S1) and primary motor (M1) signals over ROls outlined in (a). The time window of stimulus response is indicated as dotted rectangle. Depolarizing events outside of this time window are spontaneous. Bottom: Average of the evoked S1 and M1 responses ( 25 trials) at enlarged time scale. 
Cortical activity spreads from the principal barrel to the surrounding barrel field in primary somatosensory cortex (S1) and to primary motor cortex (M1) [Fig. 3(b)]. We extracted the somatosensory and motor signals in S1 and M1 cortices by integrating circular regions of interest (ROIs) of 1-mm diameter in S1 [Fig. 3(a), black circle] and M1 [Fig. 3(a), red circle] for 25 repetitions [30 s intertrial interval; Fig. 3(c), top]. This procedure yielded stimulus-induced responses with an SNR better than 5 (marked by gray dotted rectangle) as well as spontaneous depolarizing events, with similar SNR but not locked to the stimulus [outside of dotted rectangle, Fig. 3(c), top]. The onset latency of the M1 versus the S1 response is $\sim 40 \mathrm{~ms}$ [Fig. 3(c), bottom], corresponding to a velocity of propagation of 0.1 to $0.05 \mathrm{~m} / \mathrm{s}$. These observations are in agreement with earlier experiments using classical VSD imaging ${ }^{22}$ and also consistent with the view that the vibrassae sensory input to cortex in anesthetized mice is represented first in S1 and then horizontally propagates to motor areas by, at least in part, strong monosynaptic intracortical connections. ${ }^{86,87}$

Like traditional EEG recordings, the optical imaging method is strictly noninvasive and in addition provides superior spatial resolution and permits dissociation of EEG activity into genetically defined cell types. The current work conducted on mice under anesthesia is certainly an insufficient model for modulations of brain states and cannot address normal behavioral brain states including natural sleep. Therefore, the ability to record from fully awake animals and/or animals during natural sleep is indispensable. Imaging of awake visual cortex of pCAGVSFP Butterfly-electroporated, head-fixed mice on a treadmill was recently benchmarked for wide-field acquisition of cortical retinotopography and cortical sensory dynamics. Using this approach, VSFP Butterfly was shown to report electrical signals of time courses commensurate with local field potential recordings and of similar spatial structure as derived from intrinsic hemodynamic optical signals. ${ }^{84}$ The wish to further ease the use of this methodology and make it widely applicable in different laboratories has motivated the development of a knock-in transgenic mouse line, Ai78, for Cre-recombinase and tetracycline transactivator-dependent conditional expression of VSFP Butterfly-1.2 (Madisen et al., submitted). Transgenic mice expressing VSFP Butterfly-1.2 in excitatory neurons of layer 2/3 demonstrated sensory-induced VSFP signals in somatosensory, visual, and auditory cortices in response to the presentation of tactile stimuli directed to the mouse vibrassae, visual gratings or tones, respectively, with single-trial sensitivity (Madisen et al., submitted). These studies demonstrated the feasibility and relative technical ease of cell- and layer-specific transcranial cortical voltage imaging.

\section{Conclusion}

The GEVI imaging opens a new observational window to nervous system function fully complementary to optogenetic interference methods ${ }^{88,89}$ by relying on genetic tools to define the population of target cells and to drive long-term indicator expression in vivo. The complementarity with optogenetic interference methods is the key toward an all-optical implementation of in vivo electrophysiology, combining optical electrical stimulation and optical read-out of electrical responses. ${ }^{74,80,90}$ Technically, the integration of optical writing and reading of neuronal information requires a method to multiplex action control and fluorescence acquisition, either by spectral or by temporal isolation of action and excitation/emission channels.
The application of GEVI imaging is not limited to the brain and is beginning to be used in cardiac sciences. ${ }^{91-94}$ For instance, heart-specific expression of VSFP2.3 exclusively targeting either myocyte or fibroblast cells in transgenic mice was used to assess the role of these cells in cardiac infarction physiology, revealing abnormal electronic coupling between these cell types in infarction border tissue and giving rise to action potential-like potentials in fibroblast cells. ${ }^{92}$ Future engineering applications may involve the control of neuromuscular signals, interfacing nervous systems with external electronic circuits and optical screening for drugs targeting the nervous system, for instance.

While the traditional domain of voltage imaging has been the visualization of supracellular spatial-temporal structure of cortical electrical signaling, there is strong interest to achieve cellular resolution imaging to support local circuit analysis in vivo, particularly of electrical activity inaccessible to calcium imaging, as argued above. For this, we tested the VSFP Butterfly-1.2 response under two-photon excitation detecting VSFP signals from resolved pyramidal cells in brain slices and population signals in vivo. ${ }^{95}$

It took more than several decades for voltage-sensitive dye imaging starting with Merocyanine-540 to eventually arrive at the present generation of low molecular weight voltage-sensitive dyes and almost two decades to develop the current state of the art GEVIs. The fascinating aspect of the history of voltage imaging is that this development was initiated at a time where optical imaging hardware beyond the classical anatomical microscope did not yet exist. The strong hope today is that the GEVIs will add to building a powerful optical toolbox for neuronal system physiology to eventually justify the enthusiasm that has inspired this decade-long development of optical measurement of membrane voltage that began with the pioneering work of Cohen and colleagues.

\section{Acknowledgments}

We would like to thank the current members of the Knöpfel Lab for scientific advice and insightful discussions. We would also like to thank GlaxoSmithKline and the Human Frontiers Science Program for their financial support of the work conducted in our laboratory.

\section{References}

1. L. B. Cohen and B. M. Salzberg, "Optical measurement of membrane potential," Rev. Physiol., Biochem. Pharmacol. 83, 35-88 (1978).

2. B. M. Salzberg et al., "Optical recording of neuronal activity in an invertebrate central nervous system: simultaneous monitoring of several neurons," J. Neurophysiol. 40, 1281-1291 (1977).

3. B. M. Salzberg, H. V. Davila, and L.B. Cohen, "Optical recording of impulses in individual neurones of an invertebrate central nervous system," Nature 246, 508-509 (1973).

4. A. Grinvald, B. M. Salzberg, and L. B. Cohen, "Simultaneous recording from several neurones in an invertebrate central nervous system," Nature 268, 140-142 (1977).

5. H. V. Davila et al., "A large change in axon fluorescence that provides a promising method for measuring membrane potential," Nat. New Biol. 241, 159-160 (1973).

6. L. B. Cohen et al., "Changes in axon fluorescence during activity: molecular probes of membrane potential," J. Membr. Biol. 19, 1-36 (1974).

7. D. Shoham et al., "Imaging cortical dynamics at high spatial and temporal resolution with novel blue voltage-sensitive dyes," Neuron $\mathbf{2 4}$, 791-802 (1999). 
8. P. Fromherz et al., "ANNINE-6plus, a voltage-sensitive dye with good solubility, strong membrane binding and high sensitivity," Eur. Biophys. J. 37, 509-514 (2008).

9. B. Kuhn, P. Fromherz, and W. Denk, "High sensitivity of Stark-shift voltage-sensing dyes by one- or two-photon excitation near the red spectral edge," Biophys. J. 87, 631-639 (2004).

10. J. Y. Wu et al., "Voltage-sensitive dyes for monitoring multineuronal activity in the intact central nervous system," Histochem. J. 30, 169-187 (1998).

11. P. Yan et al., "Palette of fluorinated voltage-sensitive hemicyanine dyes," Proc. Natl. Acad. Sci. U. S. A. 109, 20443-20448 (2012).

12. A. Grinvald et al., "Improved fluorescent probes for the measurement of rapid changes in membrane potential," Biophys. J. 39, 301-308 (1982).

13. A. Grinvald and R. Hildesheim, "VSDI: a new era in functional imaging of cortical dynamics," Nat. Rev. Neurosci. 5, 874-885 (2004).

14. H. S. Orbach, L. B. Cohen, and A. Grinvald, "Optical mapping of electrical activity in rat somatosensory and visual cortex," J. Neurosci. 5, 1886-1895 (1985).

15. R. Y. Tsien, "New calcium indicators and buffers with high selectivity against magnesium and protons: design, synthesis, and properties of prototype structures," Biochemistry 19, 2396-2404 (1980).

16. G. Grynkiewicz, M. Poenie, and R. Y. Tsien, "A new generation of $\mathrm{Ca}^{2+}$ indicators with greatly improved fluorescence properties," J. Biol. Chem. 260, 3440-3450 (1985).

17. R. Muri and T. Knopfel, "Activity induced elevations of intracellular calcium concentration in neurons of the deep cerebellar nuclei," J. Neurophysiol. 71, 420-428 (1994).

18. T. Knopfel and B. H. Gahwiler, "Activity-induced elevations of intracellular calcium concentration in pyramidal and nonpyramidal cells of the CA3 region of rat hippocampal slice cultures," J. Neurophysiol. $\mathbf{6 8}$, 961-963 (1992).

19. T. Knopfel, J. Diez-Garcia, and W. Akemann, "Optical probing of neuronal circuit dynamics: genetically encoded versus classical fluorescent sensors," Trends Neurosci. 29, 160-166 (2006).

20. D. S. Peterka, H. Takahashi, and R. Yuste, "Imaging voltage in neurons," Neuron 69, 9-21 (2011).

21. M. H. Mohajerani et al., "Spontaneous cortical activity alternates between motifs defined by regional axonal projections," Nat. Neurosci. 16, 1426-1435 (2013).

22. I. Ferezou et al., "Spatiotemporal dynamics of cortical sensorimotor integration in behaving mice," Neuron 56, 907-923 (2007).

23. C. C. Petersen, A. Grinvald, and B. Sakmann, "Spatiotemporal dynamics of sensory responses in layer $2 / 3$ of rat barrel cortex measured in vivo by voltage-sensitive dye imaging combined with whole-cell voltage recordings and neuron reconstructions," J. Neurosci. 23, 1298-1309 (2003).

24. M. Djurisic et al., "Functional structure of the mitral cell dendritic tuft in the rat olfactory bulb," J. Neurosci. 28, 4057-4068 (2008).

25. M. A. Popovic et al., "The spatio-temporal characteristics of action potential initiation in layer 5 pyramidal neurons: a voltage imaging study," J. Physiol. 589, 4167-4187 (2011).

26. B. Kuhn, W. Denk, and R. M. Bruno, "In vivo two-photon voltage-sensitive dye imaging reveals top-down control of cortical layers 1 and 2 during wakefulness," Proc. Natl. Acad. Sci. U. S. A. 105, 7588-7593 (2008).

27. S. Mennerick et al., "Diverse voltage-sensitive dyes modulate GABAA receptor function," J. Neurosci. 30, 2871-2879 (2010).

28. T. Knopfel, "Genetically encoded optical indicators for the analysis of neuronal circuits," Nat. Rev. Neurosci. 13(10), 687-700 (2012).

29. F. Helmchen and A. Konnerth, Imaging in Neuroscience a Laboratory Manual, Cold Spring Harbor Laboratory Press, Cold Spring Harbor, New York (2011).

30. R. D. Frostig, In Vivo Optical Imaging of Brain Function, CRC Press, Boca Raton, Florida (2009).

31. M. Chalfie et al., "Green fluorescent protein as a marker for geneexpression," Science 263, 802-805 (1994).

32. A. Miyawaki et al., "Fluorescent indicators for $\mathrm{Ca}^{2+}$ based on green fluorescent proteins and calmodulin," Nature 388, 882-887 (1997).

33. V. A. Romoser, P. M. Hinkle, and A. Persechini, "Detection in living cells of $\mathrm{Ca}^{2+}$-dependent changes in the fluorescence emission of an indicator composed of two green fluorescent protein variants linked by a calmodulin-binding sequence. A new class of fluorescent indicators," J. Biol. Chem. 272, 13270-13274 (1997).
34. T. Foerster, "Experimentelle und theoretische Untersuchung des zwischenmolekularen Uebergangs von Elektronenanregungsenergie," Z. Naturforsch. A 4, 321 (1949).

35. M. S. Siegel and E. Y. Isacoff, "A genetically encoded optical probe of membrane voltage," Neuron 19, 735-741 (1997).

36. L. M. Mannuzzu, M. M. Moronne, and E. Y. Isacoff, "Direct physical measure of conformational rearrangement underlying potassium channel gating," Science 271, 213-216 (1996).

37. A. Cha and F. Bezanilla, "Characterizing voltage-dependent conformational changes in the Shaker $\mathrm{K}^{+}$channel with fluorescence," Neuron 19, 1127-1140 (1997).

38. K. Ataka and V. A. Pieribone, "A genetically targetable fluorescent probe of channel gating with rapid kinetics," Biophys. J. 82, 509-516 (2002).

39. R. Sakai et al., "Design and characterization of a DNA-encoded, voltage-sensitive fluorescent protein," Eur. J. Neurosci. 13, 2314-2318 (2001).

40. B. J. Baker et al., "Three fluorescent protein voltage sensors exhibit low plasma membrane expression in mammalian cells," J. Neurosci. Methods 161, 32-38 (2007).

41. Y. Murata et al., "Phosphoinositide phosphatase activity coupled to an intrinsic voltage sensor," Nature 435, 1239-1243 (2005).

42. M. Sasaki, M. Takagi, and Y. Okamura, "A voltage sensor-domain protein is a voltage-gated proton channel," Science 312, 589-592 (2006).

43. I. S. Ramsey et al., "A voltage-gated proton-selective channel lacking the pore domain," Nature 440, 1213-1216 (2006).

44. S. C. Kohout et al., "Subunit organization and functional transitions in Ci-VSP," Nat. Struct. Mol. Biol. 15, 106-108 (2007).

45. C. Deutsch, "Potassium channel ontogeny," Annu. Rev. Physiol 64, 19-46 (2002).

46. Y. He et al., "A fluorescent protein voltage probe based on the voltage sensing domain of Ci-VSP," Biophys. J. 330A (2007).

47. D. Dimitrov et al., "Engineering and characterization of an enhanced fluorescent protein voltage sensor," PLoS One 2, e440 (2007).

48. A. Lundby et al., "Engineering of a genetically encodable fluorescent voltage sensor exploiting fast Ci-VSP voltage-sensing movements," PLoS One 3, e2514 (2008).

49. W. Akemann et al., "Imaging brain electric signals with genetically targeted voltage-sensitive fluorescent proteins," Nat. Methods 7, 643-649 (2010).

50. H. Tsutsui et al., "Improving membrane voltage measurements using FRET with new fluorescent proteins," Nat. Methods 5, 683-685 (2008).

51. H. Mutoh et al., "Spectrally-resolved response properties of the three most advanced FRET based fluorescent protein voltage probes," PLoS One 4, e4555 (2009).

52. A. J. Lam et al., "Improving FRET dynamic range with bright green and red fluorescent proteins," Nat. Methods 9, 1005-1012 (2012).

53. S. K. Aggarwal and R. MacKinnon, "Contribution of the S4 segment to gating charge in the Shaker $\mathrm{K}^{+}$channel," Neuron 16, 1169-1177 (1996).

54. C. M. Armstrong and F. Bezanilla, "Currents related to movement of the gating particles of the sodium channels," Nature 242, 459-461 (1973).

55. C. A. Villalba-Galea et al., "Charge movement of a voltage-sensitive fluorescent protein," Biophys. J. 96, L19-L21 (2009).

56. W. Akemann et al., "Effect of voltage sensitive fluorescent proteins on neuronal excitability," Biophys. J. 96, 3959-3976 (2009).

57. A. Lundby, W. Akemann, and T. Knopfel, "Biophysical characterization of the fluorescent protein voltage probe VSFP2.3 based on the voltagesensing domain of Ci-VSP," Eur. Biophys. J. 39, 1625-1635 (2010).

58. A. Perron et al., "Red-shifted voltage-sensitive fluorescent proteins," Chem. Biol. 16, 1268-1277 (2009).

59. B. J. Baker et al., "Genetically encoded fluorescent voltage sensors using the voltage-sensing domain of Nematostella and Danio phosphatases exhibit fast kinetics," J. Neurosci. Methods 208, 190-196 (2012).

60. L. Jin et al., "Single action potentials and subthreshold electrical events imaged in neurons with a fluorescent protein voltage probe," Neuron 75, 779-785 (2012).

61. S. G. Gautam et al., "Exploration of fluorescent protein voltage probes based on circularly permuted fluorescent proteins," Front. Neuroeng. 2, 14 (2009).

62. L. Barnett et al., "A fluorescent, genetically-encoded voltage probe capable of resolving action potentials," PLoS One 7, e43454 (2012). 
63. L. Jin et al., "Random insertion of split-cans of the fluorescent protein venus into Shaker channels yields voltage sensitive probes with improved membrane localization in mammalian cells," J. Neurosci. Methods 199, 1-9 (2011).

64. J. M. Kralj et al., "Optical recording of action potentials in mammalian neurons using a microbial rhodopsin," Nat. Methods 9, 90-95 (2011).

65. Y. Gong, J. Z. Li, and M. J. Schnitzer, "Enhanced archaerhodopsin fluorescent protein voltage indicators," PLoS One 8, e66959 (2013).

66. D. Maclaurin et al., "Mechanism of voltage-sensitive fluorescence in a microbial rhodopsin," Proc. Natl. Acad. Sci. U. S. A. 110, 5939-5944 (2013).

67. H. Mutoh and T. Knopfel, "Probing neuronal activities with genetically encoded optical indicators: from a historical to a forward-looking perspective," Pflugers Arch. 465, 361-371 (2013).

68. W. Akemann et al., "Imaging neural circuit dynamics with a voltagesensitive fluorescent protein," J. Neurophysiol. 108, 2323-2337 (2012).

69. C. Stockklausner et al., "A sequence motif responsible for ER export and surface expression of Kir2.0 inward rectifier $\mathrm{K}^{+}$channels," FEBS Lett. 493, 129-133 (2001).

70. D. Ma et al., "Role of ER export signals in controlling surface potassium channel numbers," Science 291, 316-319 (2001).

71. H. Tsutsui et al., "Improved detection of electrical activity with a voltage probe based on a voltage-sensing phosphatase," J. Physiol. 591, 4427-4437 (2013).

72. A. Perron et al., "Second and third generation voltage-sensitive fluorescent proteins for monitoring membrane potential," Front. Mol. Neurosci. 2, 5 (2009).

73. G. Cao et al., "Genetically targeted optical electrophysiology in intact neural circuits," Cell 154, 904-913 (2013).

74. Y. Mishina, H. Mutoh, and T. Knopfel, "Optogenetic electrophysiology: a new approach to combine cellular and systems physiology," Biomol. Concepts 3, 193-201 (2012).

75. Y. Mishina, H. Mutoh, and T. Knopfel, "Transfer of Kv3.1 voltage sensor features to the isolated Ci-VSP voltage-sensing domain," Biophys. J. 103, 669-676 (2012).

76. Y. Mishina et al., "Exploration of genetically encoded voltage indicators based on a chimeric voltage sensing domain," Front. Mol. Neurosci. 7, 78 (2014).

77. D. R. Hochbaum et al., "All-optical electrophysiology in mammalian neurons using engineered microbial rhodopsins," Nat. Methods 11, 825-833 (2014).

78. Y. Gong et al., "Imaging neural spiking in brain tissue using FRETopsin protein voltage sensors," Nat. Commun. 5, 3674 (2014).
79. P. Zou et al., "Bright and fast multicoloured voltage reporters via electrochromic FRET," Nat. Commun. 5, 4625 (2014).

80. Y. Gong, J. Z. Li, and M. J. Schnitzer, "Enhanced archaerhodopsin fluorescent protein voltage indicators," PLoS One 8, e66959 (2013).

81. Y. Gong et al., "Imaging neural spiking in brain tissue using FRETopsin protein voltage sensors," Nat. Commun. 5, 3674 (2014).

82. F. St-Pierre et al., "High-fidelity optical reporting of neuronal electrical activity with an ultrafast fluorescent voltage sensor," Nat. Neurosci. 17, 884-889 (2014).

83. W. Akemann et al., "Imaging neural circuit dynamics with a voltagesensitive fluorescent protein," J. Neurophysiol. 108, 2323-2337 (2012).

84. M. Carandini et al., "Imaging the awake visual cortex with a geneticallyencoded voltage indicator," J. Neurosci., in press (2015).

85. G. Scott et al., "Voltage imaging of waking mouse cortex reveals emergence of critical neuronal dynamics," J. Neurosci. 34, 16611-16620 (2014).

86. T. W. Chen et al., "Ultrasensitive fluorescent proteins for imaging neuronal activity," Nature 499, 295-300 (2013).

87. T. R. Sato and K. Svoboda, "The functional properties of barrel cortex neurons projecting to the primary motor cortex," J. Neurosci. 30, 42564260 (2010)

88. E. S. Boyden et al., "Millisecond-timescale, genetically targeted optical control of neural activity," Nat. Neurosci. 8, 1263-1268 (2005).

89. L. Fenno, O. Yizhar, and K. Deisseroth, "The development and application of optogenetics," Annu. Rev. Neurosci. 34, 389-412 (2011).

90. D. R. Hochbaum et al., "All-optical electrophysiology in mammalian neurons using engineered microbial rhodopsins," Nat. Methods 11, 825-833 (2014).

91. L. Kaestner and P. Lipp, "Screening action potentials: the power of light," Front. Pharmacol. 2, 42 (2011).

92. T. A. Quinn et al., "Cell-specific expression of voltage-sensitive protein confirms cardiac myocyte to non-myocyte electrotonic coupling in healed murine infarct border tissue," Circulation 130, A11749 (2014).

93. J. H. Hou et al., "Simultaneous mapping of membrane voltage and calcium in zebrafish heart in vivo reveals chamber-specific developmental transitions in ionic currents," Front. Physiol. 5, 344 (2014).

94. J. S. Leyton-Mange et al., "Rapid cellular phenotyping of human pluripotent stem cell-derived cardiomyocytes using a genetically encoded fluorescent voltage sensor," Stem Cell Rep. 2, 163-170 (2014).

95. W. Akemann et al., "Two-photon voltage imaging using a genetically encoded voltage indicator," Sci. Rep. 3, 2231 (2013).

Biographies of the authors are not available. 